\title{
FIB Method of Sectioning of III-V Core-Multi-Shell Nanowires for Analysis of Core/Sell Interfaces by High Resolution TEM
}

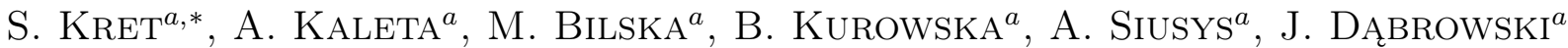 \\ AND J. SADOWSKI ${ }^{a, b, c}$ \\ ${ }^{a}$ Institute of Physics, Polish Academy of Sciences, Al. Lotnikow 32/46, PL-02668 Warsaw, Poland \\ ${ }^{b}$ MAX-IV laboratory, Lund University, P.O. Box 118, SE-221 00 Lund, Sweden \\ ${ }^{c}$ Department of Physics and Electrical Engineering, Linnaeus University, SE-351 95 Växjö, Sweden
}

\begin{abstract}
The core-multishell wurtzite structure (In,Ga)As-(Ga,Al)As-(Ga,Mn)As semiconductor nanowires have been successfully grown on GaAs(111)B substrates using MBE technique. The nanowires cores were grown with gold eutectic catalyser in vapour-liquid-solid growth mode. The double shell overgrowth, on the side facets of nanowires, was performed using lower substrate temperature (about $400^{\circ} \mathrm{C}$, and $230^{\circ} \mathrm{C}$, for $(\mathrm{Ga}, \mathrm{Al}) \mathrm{As}$, and $(\mathrm{Ga}, \mathrm{Mn}) \mathrm{As}$ shell growth, respectively). The polytypic ordering, defects, chemistry and geometric perfection of the core and the shells have been analysed at atomic level by advanced transmission electron microscope techniques with the use of axial and longitudinal section of individual nanowires prepared by focused ion beam. High quality cross-sections suitable for quantitative transmission electron microscope analysis have been obtained and enabled analysis of interfaces between the core and the shells with near atomic resolution. All investigated shells are epitaxial without misfit dislocations at the interface. Some of the shells thicknesses are not symmetric, which is due to the shadowing effects of neighbouring nanowires and directional character of the elemental fluxes in the MBE growth process.
\end{abstract}

DOI: 10.12693/APhysPolA.131.1332

PACS/topics: nanowires, HRTEM, STEM, EDS, FIB, GaAs, GaAlAs

\section{Introduction}

Semiconductor nanowires (NWs) are considered as a base for an emerging class of new optoelectronic and photonic nanodevices [1]. The pseudo-one-dimensionality of NWs offers a unique opportunity to control sizedependent and structure-dependent material properties for novel device functionalities. The use of core-shell NW architectures, composed of ternary III-V compound semiconductor alloys is of particular interest and provides further freedom to tune the material bandgap [2]. Nanowires grown in the core-shell geometry attract a great interest because of possible applications as building blocks of different devices, such as solar cells [3], high electron mobility transistors [4], light emitting diodes [5], and lasers [6]. The presence of coating shells improves considerably the optical and carrier transport properties of NWs due to the passivation of surface states [7]. Modern crystal growth techniques such as molecular beam epitaxy (MBE) provide a feasible way for material engineering by implementing various material combinations together with well-controlled doping levels in individual NWs.

The core of NWs can be grown by MBE using principles of the old mechanism of catalytic growth of silicon whiskers of micrometer dimensions demonstrated by Wagner and Ellis in 1965 [8]. This method is known

*corresponding author; e-mail: kret@ifpan.edu.pl as a vapour-liquid-solid (VLS) mechanism with the use of a catalyst in the form of nanodroplets. Epitaxial shells can be grown by lowering the substrate temperature which results in solidification of the catalyst droplet and blocking a long range surface diffusion. Thus epitaxial shells with different composition than that of the core can be grown on the side wells of the NW core. This method was successfully applied e.g., to grow optically active $\mathrm{ZnTe} / \mathrm{ZnMgTe} \mathrm{NWs}$ [7] or wurtzite (In,Ga)As$(\mathrm{Ga}, \mathrm{Mn}) \mathrm{As}$ core-shell NWs [9]. Investigations of the structural perfection of the interfaces between the core and the shell, which can have different elemental compositions and different lattice parameters are crucial for understanding their properties. The best method enabling investigations of NW defects and imperfections of the core-shell geometry is inspection of longitudinal and perpendicular cross-sections by transmission electron microscopy. This work presents advantages and artefacts related to different approaches of focused ion beam (FIB) preparation of high quality sections suitable for high resolution transmission electron microscopy (HRTEM) investigation.

\section{Experimental}

Nanowires were grown by the MBE method employing VLS growth mode. Prior to the growth of the nanowires a 2 Athick gold layer was deposited on epiready $\mathrm{GaAs}(111) \mathrm{B}$ substrate at low temperature $\left(100^{\circ} \mathrm{C}\right)$ in the MBE system dedicated for metals. Then the substrate was transferred (in air) to the III-V semiconductor MBE system and annealed at $590{ }^{\circ} \mathrm{C}$ to desorb the native oxide from GaAs substrate and to create small 
nanometer-scale $\mathrm{Au}-\mathrm{Ga}$ eutectic catalysts droplets on it. Core-shell nanowires were grown in two steps. In the first step, (In,Ga)As primary cores were grown at high temperature (growth temperature about $500^{\circ} \mathrm{C}$ ). The nanowires are oriented vertically with respect to the substrate surface; they are up to several micrometers long and have diameters of 70-100 $\mathrm{nm}$. In the second step, the substrate temperature was reduced and (In, Ga)As, (Al,Ga)As and/or LT-GaAs and/or LT-(Ga,Mn)As shells were deposited on the side walls of primary (In, Ga)As nanowires.

The FIB sections were prepared with the use of FEI Helios Nanolab 600 dual beam FEG-SEM/FIB apparatus equipped with Omniprobe nanomanipulator and platinum gas injection system (GIS). TEM investigations were performed by FEI Titan cube 80-300 microscope operating at $300 \mathrm{kV}$ in scanning mode TEM (STEM) at different camera lengths. Also energy-dispersive X-ray spectroscopy (EDS) investigations were performed using nanobeam electron in STEM mode.

\section{Results and discussion}

\subsection{NW cross-section by FIB sectioning method 1}

In the case of (In, Ga)As /(Ga,Mn)As core shell NWs, two alternative FIB methods are applied. In the first one, the specimen preparation procedures were applied as described below. First NWs were transferred to the surface of $\mathrm{Si}(100)$ wafer. After selection of suitable NWs and marking their position, substrate and NWs were capped with $0.2 \mu \mathrm{m}$ and $1 \mu \mathrm{m}$ thick platinum layer deposited with electrons and Ga ions, respectively. During deposition of platinum by electrons, the $5 \mathrm{kV}$ acceleration voltage and $2.7 \mathrm{pA}$ current were used. In the case of gallium ions, deposition of platinum was performed at $30 \mathrm{kV}$ and $280 \mathrm{pA}$. Final thinning was realized with $\mathrm{Ga}$ ions at $2 \mathrm{kV}$ and $28 \mathrm{pA}$. The whole process is illustrated in diagram shown in Fig. 1a. An example of a suc-
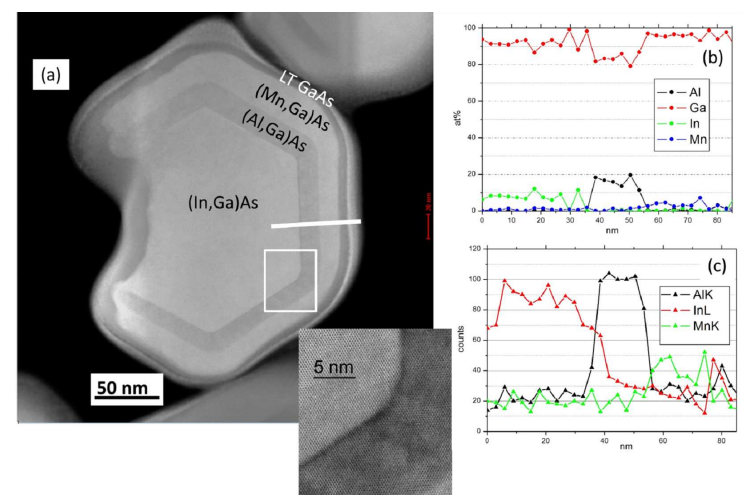

Fig. 1. (a) HR-STEM-HAADF (In,Ga)As/(Al,Ga)As $/(\mathrm{Ga}, \mathrm{Mn}) \mathrm{As} / \mathrm{LT}$ GaAs multi-shell NWs in the finally thinned lamella. The size amorphization due to the side ion interaction is visible. The part of the image in the frame is zoomed showing structural details of the interface between (In, Ga)As core and first (Al, Ga)As shell. (b) EDS quantitative profile of the elemental distribution in the core and the shells. (c) Intensities of the $\mathrm{Al}$ $K$, In $L$, and Mn $K$ line in the EDS spectra.
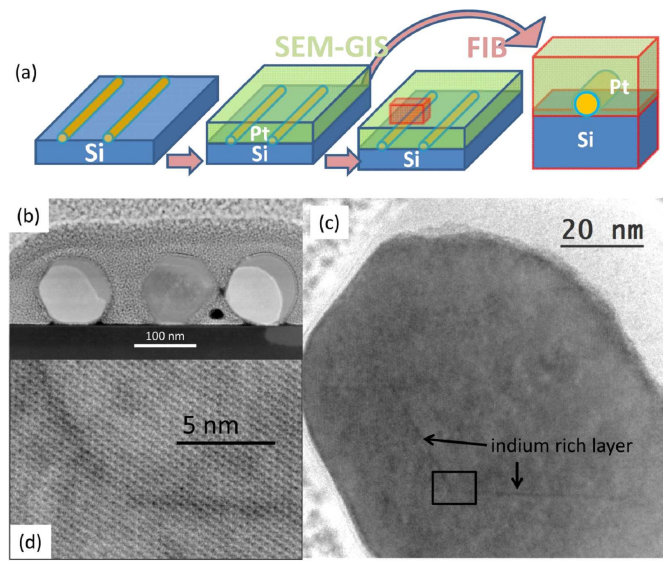

Fig. 2. (a) A diagram showing steps and geometry of the FIB cross-section of (In, Ga)As/(Ga,Mn)As NWs transferred to the surface of silicon wafer, (b) STEM image of FIB lamella showing cross-section of three NWs covered by GIS platinum deposited by electrons and by Ga ions. (c) HRTEM image showing amorphization occurring during the deposition of platinum caused by the high current of electrons. Image in the black frame is zoomed (d) showing the $0.5 \mathrm{~nm}$ thick indium enriched layer on the surface of the (In, Ga)As core.

cessful cut containing three NWs is shown in the STEM image in Fig. 1a. Parts of the NW images appearing as darker emerge, when the beam is aligned with a crystalline axis of nanowires. NWs were partially amorphised from one side due to the high current used during platinum deposition by electrons (Fig. 2b,c). Sharp interface between (In, Ga)As core and (Ga,Mn)As shell can be resolved as a dark line in HRTEM image (see Fig. 2c,d). Other EDS investigations (not shown) indicate that the interface region is enriched with indium, which is related to the transport of atoms on the NW side walls during the growth of the (In,Ga)As core. Lowering the temperature "freezes" the indium at the surface of the core. No defects were detected; the interface between the core and the shell is totally coherent. EDS investigations show that the composition of these amorphised areas does not differ in comparison to the crystalline parts.

\subsection{NW cross-section by FIB sectioning method 2}

The NWs can easily fall "mechanically", as shown in Fig. 3a. On such fallen, almost parallel bunch of NWs the protective platinum layer was deposited. Only upper NWs were glued together during such deposition. After ion milling of trenches on the both borders of lamella, before the lift-off process additional layer of platinum was deposited on two sides of the lamella (see Fig. 3b). A pre-thinned lift-off lamella attached to the $\mathrm{Cu}$ grid is shown in Fig. 3c. Such twice, double side deposited platinum, effectively protects against fragmentation of NWs containing lamella and enables continuation of the final thinning. However, the close inspection of such lamella clearly shows the existence of void between NWs, as shown in Fig. 3d. In this figure, different layers of platinum deposited during different stages can be identified as separated layers. The typical STEM-high angle annu- 
lar dark field (STEM-HAADF) image of finally thinned lamella is presented in Fig. 3e. Only the one cross-section of NW is oriented. It is due to fact that NWs in the bunch are not parallel to each other. Additional disadvantage of such a method is related to the presence of voids in the platinum "glue". During the final thinning the amorphization of the side of the NW directly exposed to the gallium beam can be observed, as shown in Fig. 3a (the incident angle is locally almost $90^{\circ}$ at the non-protected side of NWs, due to the presence of voids in the platinum deposit). The high quality of remaining part of NW cross-section enables a high resolution characterization. The individual shells are clearly visible with sharp interfaces at the atomic level. The quality of EDS spectra obtained from such cross-section enables getting the qualitative, but also quantitative profiles of the elemental distribution in the core and in the shells (see Fig. 1b).

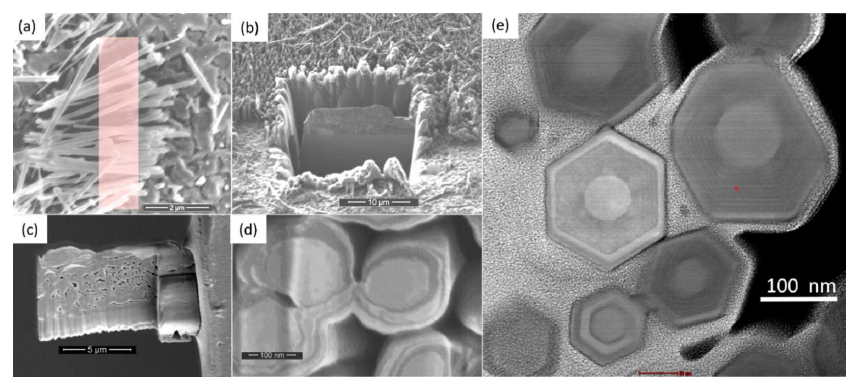

Fig. 3. (a) SEM image of a fallen (In,Ga)As/(Al,Ga)As/(Ga,Mn)As/LT GaAs multishell NWs with schematic position of the platinum deposition region. (b) Ion microscopy image of a lamella after the side deposition of platinum before the lift-off. (c) SEM image of the lamella before the final thinning (d) magnified SEM image of the cross-section of NWs with different layers of platinum deposition, (e) STEM-HAADF image of (In, Ga)As/SL-(Ga,Al)As core-shell after final thinning.

Analysis of the elemental composition of the shells by EDS shows that the concentrations of $\mathrm{Al}$ and $\mathrm{Mn}$ in relation to gallium/with the reference to $\mathrm{Ga}$ are 18 and 5 at.\%, respectively; which matches well the MBE calibrations performed for planar growth [10] with the same elemental flux intensities as used for the growth of NWs. Such a good agreement permits us to believe that our EDS measurements of the indium content in the shells reach the level of $8-9$ at.\%. This quantification is possible due to the final $2 \mathrm{kV}$ final cleaning of lamella to minimize the incorporation of gallium to side wells. The literature data concerning simulations of Ga atoms implantation shows that during FIB cleaning at $5 \mathrm{kV}$, the penetration depth into silicon is about $4 \mathrm{~nm}$, with maximum atomic concentration of 2 at.\% [11, 12]. Thus, in the case of performing $2 \mathrm{kV}$ final cleaning, implantation of gallium can be neglected for EDS quantification when 30-50 nm lamella is used. This information cannot be obtained by other means. The VLS growth mechanism is different from a 2D epitaxial growth, so incorporation of indium can be different.
Our results show that the concentration scaling based on XRD analysis of 2D layers growing together with NW are not proper for estimation of elements concentration into nanowire during axial growth, however they are valid for radial growth of the shells. Moreover, because of the presence of the shells, a quantitative elemental analysis of the core is impossible for non-sectioned NWs, due to the projection effects and averaging of information from the core and the shells. HR-STEM image (see zoomed part of Fig. 1a) clearly shows the $1 \mathrm{~nm}$ wide layer with the dark contrast corresponds to the higher aluminum concentration. Contrary to (In,Ga)As/( Ga,Mn)As NWs the enrichment of indium is not observed in this case. This can be explained by differences in the grow temperature of the core. In the case of the first sample temperature was lowered up to $220^{\circ} \mathrm{C}$ which freezes the indium on the surface of the core. In the case of second sample the grow temperature of (Al, Ga)As shell was almost the same as temperature of the core.

\subsection{Longitudinal FIB section of NWs}

We have tried to apply the same standard procedure of FIB lamella preparation which is usually applied to epitaxial films. During deposition of a protective platinum layer, small nanocrystals of platinum were deposited on the surface of NWs up to the substrate, which created a 10-20 nm thick cover on each NW. However, the space between them was not filled (see Fig. 4a). Continuous platinum layer reaches maximum $2 \mu \mathrm{m}$ of depth from the top and does not reach bottom parts of the $4-6 \mu \mathrm{m}$ long NWs. The presence of voids between the NWs results in channeling of the ions and selective etching. After thinning of the lamella, sectioned from the one side NWs were covered from the second side with the layers of nanocrystalline platinum giving a strong contribution to the image contrast, which made interpretation of TEM/STEM images impossible or difficult (see Fig. 4b). In Fig. 4c, the HR-STEM image is shown, but the structural image of the crystal lattice of NWs cannot be clearly resolved here.

The simple method to solve this problem is embedding the NWs by putting droplet of an epoxy glue on a surface, prior the standard FIB cross-sectioning procedure. This modification permits to obtain high quality longitudinal section of NWs. Now, the individual shell thickness variations are clearly visible along the NWs length, as shown in Fig. 5b. We did not find any misfit dislocations along the whole NWs length at the interfaces between the core and the shells. However stacking faults which are present in the core propagate into the shell. This proves a perfect epitaxy of the shells. High quality HR-STEM images obtained from the longitudinal FIB sections permit to analyze the variation of the crystalline structure polymorphism along the NW axes. The bottom parts of NWs have usually wurtzite $(2 \mathrm{H})$ structure with average SF distance in the range 10 to $40 \mathrm{~nm}$. In the upper part, the density of $\mathrm{SF}$ is higher. Also a short, 3-5 monolayer segments of a cubic material (3C) is observed there (not shown). Moreover, in the top-most part of NWs, the $4 \mathrm{H}$ 


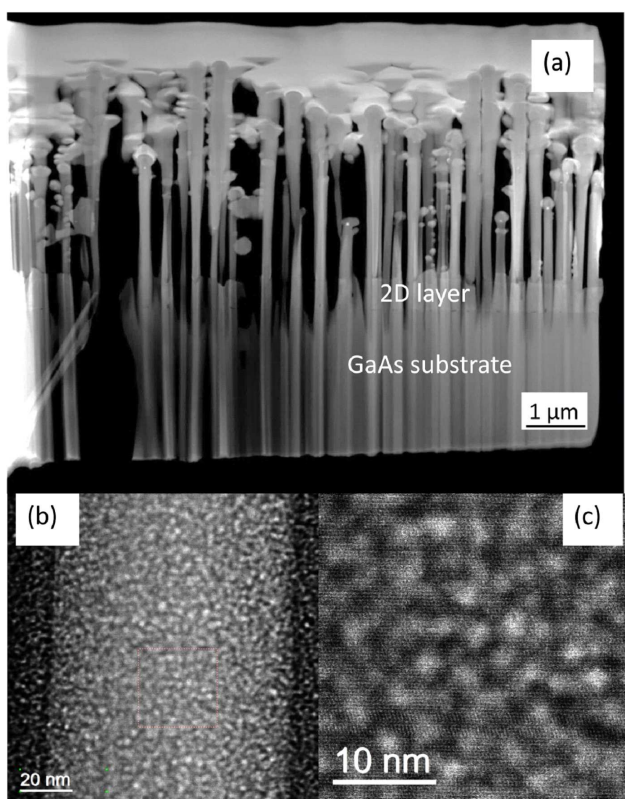

Fig. 4. (a) STEM-HAADF image of a longitudinal FIB section of NWs with direct deposition of platinum on the top of NWs. (b) STEM-HAADF image of NW section covered on one side by platinum (c) HR-STEM image of zoomed part of (b) - the high contrast of platinum grains overlaps with the NW making image difficult to interpretation.

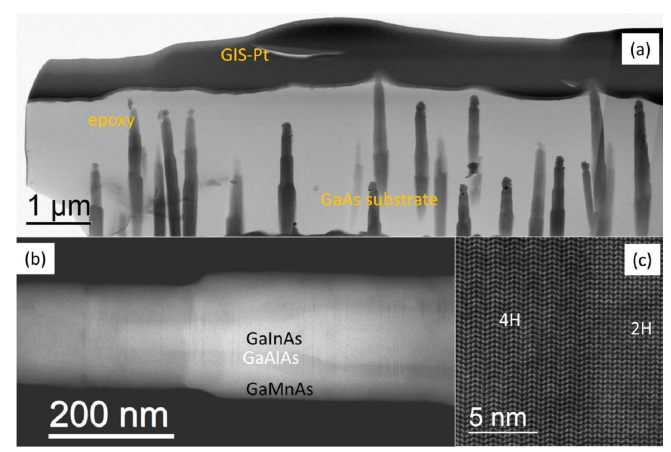

Fig. 5. (a) STEM-BF image of a longitudinal FIB section of NWs embedded in the epoxy glue prior to the deposition of platinum on the top of NWs (b) HR-STEM-HAADF image of $(\mathrm{In}, \mathrm{Ga}) \mathrm{As} /(\mathrm{Al}, \mathrm{Ga}) \mathrm{As} /(\mathrm{Ga}, \mathrm{Mn}) \mathrm{As} / \mathrm{LT}$ GaAs multishell NW showing the shape and the thickness variation of $(\mathrm{Al}, \mathrm{Ga}) \mathrm{As}$ and $(\mathrm{Ga}, \mathrm{Mn}) \mathrm{As}$ shells along the NWs axis. (c)The atomic resolution HAADF-images of NWs showing the interface between $4 \mathrm{H}$ polytype and $2 \mathrm{H}$ (Wurtzite) part of the NW.

polytypes of up to $10 \mathrm{~nm}$ long segments were observed (see Fig. 5c).

\section{Conclusions}

The sectioning of NWs is necessary to access the distribution of elements in the core and the shells in multi-shell NWs and study interfaces between the core and the shell with atomic resolution. FIB preparation permits to obtain the sections suitable to high resolution investigations. A few mono-layer enrichments of the core surface in indium and in aluminum were detected from HR-STEM images. In the case of $(\mathrm{In}, \mathrm{Ga}(\mathrm{As} /(\mathrm{Ga}, \mathrm{Mn}) \mathrm{As}$ NWs, indium enrichment on the NWs surfaces is related to freezing of the indium atoms on the surface due to lowering the growth temperature prior the shell overgrowth. In the case of (In,Ga)As/(Al,Ga(As/(Ga,Mn)As/LT GaAs core-multishell NWs, the enrichment of (In,Ga)As core surface with aluminum can be related to $\mathrm{Al}$ segregation during the epitaxial growth. For longitudinal cross-sections the NWs should be embedded in the epoxy glue prior the FIB milling procedure, in order to avoid the overlapping of heavy platinum particles with the sectioned NWs. By following such a procedure, the high quality sections can be obtained permitting atomic sale visualization of the interfaces and determination of polytypism.

\section{Acknowledgments}

This work was supported by NCN grants no. 2014/13/B/ST3/04489 and 2016/21/B/ST5/03411. The TEM investigations were performed using equipment founded by European Regional Development Fund through the Innovative Economy grant No. POIG.02.0100-14-032/08.

\section{References}

[1] Y. Li, F. Qian, J. Xiang, C.M. Lieber, Mater. Today 9, 18 (2006).

[2] C. Chen, S. Shehata, C. Fradin, R.R. LaPierre, C. Couteau, G. Weihs, Nano Lett. 7, 2584 (2007).

[3] B. Tian, X. Zheng, T.J. Kempa, Y. Fang, N. Yu, G. Yu, J. Huang, C.M. Lieber, Nature 449, 885 (2007).

[4] X. Jiang, Q. Xiong, S. Nam, F. Qian, Y. Li, C.M. Lieber, Nano Lett. 7), 3214 (2007).

[5] F. Qian, S. Gradecak, Y. Li, C.Y. Wen, C.M. Lieber, Nano Lett. 5, 2287 (2005).

[6] F. Qian, Y. Li, S. Gradecak, H.G. Park, Y.J. Dong, Y. Ding, Z.L. Wang, C.M. Lieber, Nature Mater. 7, 701 (2008).

[7] P. Wojnar, M. Szymura, W. Zaleszczyk, Ł. Kłopotowski, E. Janik, M. Wiater, L.T. Baczewski, S. Kret, G. Karczewski, J. Kossut, T. Wojtowicz, Nanotechnology 24, 365201 (2013).

[8] R.S. Wagner, W.C. Ellis, Appl. Phys. Lett. 4, 89 (1964).

[9] A. Siusys, J. Sadowski, M. Sawicki, S. Kret, T. Wojciechowski, K. Gas, W. Szuszkiewicz, A. Kamińska, T. Story, Nano Lett. 14, 4263 (2014).

[10] P. Laukkanen, J. Sadowski, M. Guina, Semicond. Res. Springer Series Mater. Sci. 150, 1 (2012).

[11] K. Thompson, B. Gorman, D.J. Larson, Brandon van Leer, Liang Hong, Microsc. Microanal. 12, 1736 (2006).

[12] D.A. Cullen, D.J. Smith, J. Appl. Phys. 104, 094304 (2008). 